\title{
Desarrollo de estrategia colectiva indígena: una visión teórica y práctica desde la gestión moderna para el desarrollo territorial aymara
}

\author{
Indigenous collective strategy development: a theoretical and practical view from modern \\ management for aymara territorial development
}

Rodrigo Ignacio Barra Novoa*

\begin{abstract}
Resumen: Los pueblos indígenas de Chile enfrentan muchos desafíos locales y comunitarios debido a la globalización, el cambio tecnológico y una economía de mercado neoliberal con poco apoyo gubernamental. En este sentido, varios pueblos indígenas continúan viviendo en malas condiciones y pobreza. Sin embargo, el liderazgo colectivo ha demostrado ser una herramienta eficaz para abordar diversas fallas de mercado y barreras institucionales que obstruyen su desarrollo cultural, social y económico a nivel local. Este artículo analiza los principales postulados teóricos y prácticos para que las estrategias colectivas lideradas por comunidades indígenas puedan ser desarrolladas y desplegadas de manera eficiente en su contexto territorial y patrimonial.
\end{abstract}

Palabras clave: Comunidades indígenas, estrategia colectiva, crecimiento social y cultural, desarrollo económico.

Abstract: Chile's indigenous peoples face many local and community challenges due to globalization, technological change, and a neoliberal market economy with little government support. In this sense, several indigenous peoples continue to live in poor conditions and poverty. However, collective leadership has proven to be an effective tool to address the various market failures and institutional barriers that obstruct its cultural, social and economic development at the local level. This article analyzes the main theoretical and practical postulates so that the collective strategies led by indigenous communities can be developed and deployed efficiently in their territorial and patrimonial context.

Keywords: Indigenous communities, economic development, collective estrategic, social and cultural growth.

Recibido: 17 abril 2021 Aceptado: 7 junio 2021

\footnotetext{
* Doctorando en Ciencias Jurídicas y Económicas por la Universidad Camilo José Cela - España. Máster Oficial en Estrategias Territoriales y Ambientales en el Ámbito Local por la Universidad de Huelva - España Asesor Especialista Centro de Negocios Sercotec Arica. Programa ejecutado por la Universidad de Tarapacá. rodrigo.barra.novoa@gmail.com / www.centroschile.cl
} 


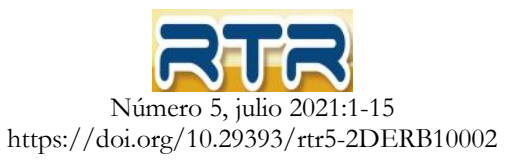

\section{Introducción}

Los pueblos indígenas de Chile enfrentan diversos desafíos locales y comunitarios debido a la globalización, el cambio tecnológico y una economía de mercado neoliberal con poco apoyo gubernamental. En este sentido, varios pueblos indígenas continúan viviendo en malas condiciones y pobreza. Sin embargo, el liderazgo colectivo ha demostrado ser una herramienta eficaz para abordar las diversas fallas de mercado y las barreras institucionales que impiden su desarrollo cultural, social y económico en el ámbito local.

La revisión de la literatura internacional indica que el liderazgo y la capacidad de organización moderna requiere de la restauración del liderazgo indígena, así como de la adopción y transferencia de conocimiento ancestral entre los líderes más longevos y los más modernos. Además, los líderes y sus organizaciones al tener que adaptarse a los rápidos cambios que están ocurriendo, deben satisfacer las necesidades y aspiraciones de sus comunidades, al tiempo que preservan su patrimonio e identidad local.

Diversos enfoques de desarrollo económico y equilibrio estático impuestos bajo la política de regionalización impulsada en el gobierno militar, perseguían especialmente en las zonas de precordillera y altiplano de la Provincia de Parinacota, la soberanía en estos espacios fronterizos y contribuir al arraigo de su población, dotándola de incentivos de conectividad e infraestructura con un marcado aparato burocrático estatal en el territorio que hasta la fecha ha tenido una contribución muy modesta en el desarrollo económico de las comunidades indígenas.

Esto último, ha sido reconocido recientemente por los agentes institucionales de la región, buscando asumir nuevas estrategias y metodologías de trabajo que afiancen internamente procesos de desarrollo en la población local, orientado preferentemente a la gestión de los recursos productivos, humanos, culturales y medioambientales, prácticamente lo único con que se cuenta para su desarrollo.

Considerando lo anterior, el desarrollo no puede excluir a ningún grupo social, al menos debe garantizarse la existencia de alternativas de desenvolvimiento para la población, por lo que las comunidades y líderes locales han despertado paulatinamente sus inquietudes de una renovación y estrategia colectiva para sus organizaciones y comunidades, que cobra relevancia como medio reivindicativo para una mejor calidad de vida en sus territorios.

\section{Revisión de literatura}

En términos generales, la expansión del tema del desarrollo indígena está obligando a los agentes públicos y privados a adoptar herramientas del sector empresarial y aplicarlas dentro de las líneas de fortalecimiento organizacional para lograr un mejor desempeño en las actividades comerciales y culturales de las comunidades indígenas en Chile. De acuerdo con este postulado, la idiosincrasia, el contexto cultural y las prácticas ancestrales de gestión comunitaria, reconocen la importancia de la sabiduría, el liderazgo y las historias propias de la comunidad para lograr el éxito organizacional, lo que implica un proceso de cooperación, relaciones e intercambios duraderos (Davis, 1997; Weber \& Khademian, 2008; Popp, 2013 et al.,).

Si bien no es objetivo de este documento llegar a un consenso sobre todos los postulados políticos, sociales y operativos de la estrategia colectiva, su ejecución implica un conjunto de rutinas, procedimientos y reglas. 
La premisa general, aunque no excluyente, indica que la estrategia colectiva requiere una correcta planificación, vinculando objetivos, indicadores y metas para llevar a cabo con éxito una adecuada implementación estratégica.

En este sentido, la eficiencia de la estrategia colectiva está relacionada con la cercanía de la organización a su frontera de gestión y liderazgo eficiente. Si la organización está muy bien gestionada probablemente sea el resultado de una correcta aplicación de herramientas como el Balanced Scorecard (BSC), o una eficiente administración de los recursos y capacidades de la organización (Penrose, 1959).

Además, las sinergias operativas y sociales también son factores estratégicos que podrían justificar la extensión del liderazgo y la integración de diferentes grupos de interés. Estas sinergias explican, al menos en parte, la provisión de mejoras internas, así como el surgimiento de la búsqueda de un mejor poder de negociación entre las comunidades locales y los agentes institucionales del territorio.

Existen otras posibles fuentes de creación de valor territorial a partir de la integración de todos los elementos del sistema de gestión estratégica, que según Kaplan y Norton (1993) alinean la misión de la estrategia con el conjunto de medidas clave de desempeño en torno a cuatro perspectivas: procesos internos, clientes, finanzas, aprendizaje y crecimiento.

Este marco proporciona equilibrio a los resultados que la organización necesita lograr. Por lo tanto, el razonamiento de causa y efecto de la estrategia colectiva, especialmente para el contexto territorial indígena, invita a los responsables de políticas de desarrollo local a reflexionar sobre el pensamiento estratégico y el liderazgo para crear valor, especialmente para apoyar la implementación de temas colectivos.

Autores como Peter Drucker ${ }^{1}$, Kenneth Andrews ${ }^{2}$, Alfred Chandler ${ }^{3}$ e Igor Ansoff, identifican la necesidad de pensar la estrategia colectiva de forma articulada y dinámica, que unifica las ideas individuales de cada una de las áreas funcionales de la organización y las relaciona con el entorno político, social y competitivo en el que se desenvuelve.

Teniendo en cuenta lo anterior, en el componente de resultados del análisis conceptual se explican algunos elementos teóricos y prácticos de la literatura sobre el desarrollo de estrategias colectivas, que incluye componentes clave como el análisis de las necesidades políticas, sociales y éticas de las organizaciones; metodologías para la evolución de las estrategias colectivas y sus oportunidades futuras; identificación de oportunidades para incrementar el impacto de las estrategias en las partes interesadas; definición de los grupos de interés que componen la organización; y una serie de insumos y conocimientos comunicacionales, estratégicos y administrativos para lograr los propósitos de la estrategia organizacional colectiva.

\section{Metodología}

Desde el punto de vista metodológico, el artículo es de tipo explicativo, descriptivo y comprensivo, que analiza las principales aportaciones de la gestión estratégica moderna para conseguir un adecuado desarrollo de una estrategia colectiva indígena. La investigación examina factores cualitativos y explica una serie de ámbitos políticos, sociales, éticos y operativos que ayudan a exponer los alcances de una

${ }^{1}$ Drucker, P 1954. La práctica de la gestión. Harper, Nueva York.

${ }^{2}$ Kenneth A. 1971. El concepto de estrategia corporativa. Irwin, Homewood, III.

${ }^{3}$ Chandler, A. 1962. Estrategia y estructura. The MIT Press, Cambridge, MA. 
adecuada estrategia. Además, se contextualiza el rol de los actores locales, que ayudan a explicar la importancia entre los agentes involucrados para potenciar el impacto en las organizaciones, comunidades y emprendimientos asociativos indígenas.

Adicionalmente, se opta por examinar una serie de artículos, documentos, estadísticas y consulta a expertos, considerando la escasa sistematización de este tipo de investigaciones en la Región de Arica y Parinacota.

\section{Resultados del análisis conceptual}

\section{Análisis de las necesidades políticas, éticas y operativas de las estrategias comunes entre organizaciones y administraciones}

Durante décadas, una doctrina organizacional ha dominado el escenario estratégico entre ejecutivos y departamentos de liderazgo: Maximizar el valor para los accionistas (MSV). A lo largo de los últimos años ha generado controversia, con opiniones tan polarizadas que es una herramienta aspiracional o absoluta para medir el desempeño de la gerencia (Clarke \& Friedman, 2016). Sin embargo, probablemente por estas visiones contrastadas y la evolución de las necesidades del mercado en un mundo con cambios extremos, el concepto de MSV se ha vuelto casi obsoleto. Ahora no solo importa aumentar la riqueza de los accionistas sino también el bienestar social. En la historia, como explica Clarke \& Friedman (2016), la MSV ha demostrado que podría perjudicar a la sociedad por varios motivos, algunos de ellos por subestimar las acciones del mundo indígena y la falta de interés en ayudar al desarrollo con impacto.

El mundo está cambiando, para bien o para mal, y los empresarios, especialmente de origen étnico, no suelen pensar profundamente en las implicaciones que esto conlleva para sus empresas u organizaciones (Kourdi, 2010). En detalle, Verweire (2014) da un ejemplo estadístico sobre esta aplicación incorrecta de la estrategia. El alcance es que las tasas de fallas oscilan entre el 40\% y el 90\%, lo que es realmente alto. En consecuencia, surgen necesidades fundamentales en las áreas política, social, ética y operativa para ayudar en la construcción de estrategias para prácticas organizacionales más consistentes para el mundo y cosmovisión indígena.

$\mathrm{Al}$ explicar las necesidades políticas para una aplicación efectiva de la estrategia, la literatura enfatiza la importancia de que el Derecho reconozca a las organizaciones como seres humanos, ya que muchas de ellas no actuaron socialmente años antes, lo que es una preocupación común en la política internacional. De hecho, Greenfield a través de Clarke \& Friedman (2016) abordó esta visión y afirmó que esto daría constitucionalmente el derecho a pedir a las empresas que se comporten como personas decentes y éticas.

Esto se vuelve importante para poder hacer una estrategia empática. Todos los partidos políticos y las organizaciones territoriales necesitan transformar su forma de gobernar (Barber, 2005). Para lograr esto, Barber encontró seis impulsores clave: liderazgo comunitario, regulación y monitoreo, escrutinio y desafío, habilidades de comunicación, trabajo en asociación y entendimiento político.

Socialmente, la estrategia podría basarse en la inversión de capital, tomando parte de las ganancias en beneficio y valor humano, y priorizando la satisfacción de la sociedad y los grupos de interés (Clarke \& Friedman, 2016) También es importante crear una agenda climática según Hoffman (2014). Hoy en día, el $85 \%$ de los empresarios coinciden en que el cambio climático es un factor humano en contraste con 
el $62 \%$ y el $76 \%$ de la población en general que lo hace también, pero con casi la mitad o un tercio que aún no lo hace (Hoffman, 2014). Éticamente, tiene que haber un enfoque de liderazgo, gestión y una responsabilidad hacia las partes interesadas en general. En el caso de las operaciones, hay varios escenarios a considerar: por ejemplo, en el área de M\&A, es valioso pensar que las (sub) culturas no son estáticas ni fijas, sino que son construcciones sociales dinámicas que juegan un papel en la integración (Van Marrewijk, 2016). En el caso de la transformación digital, una visión de asociarse con terceros (por ejemplo, startups) es parte de un largo viaje de adaptación para la creación de productos innovadores (Birkinshaw, et al, 2016). Pero lo que todos deberían construir como prioridad es una visión de largo plazo (Clarke \& Friedman, 2016) y tener plena certeza de su propuesta de valor, modelo operativo y mercado (Verweire, 2014).

\section{Metodologías de estrategias colectivas para el desarrollo dentro de una organización y externamente sobre una base colaborativa}

Lograr con éxito los requisitos antes mencionados en los ámbitos político, social, ético y operativo no es tarea fácil. Homkes (2016) menciona que entre dos tercios y tres cuartos de las empresas no logran obtener los resultados esperados de sus estrategias. Los resultados a largo plazo y el cambio de paradigma requieren un modelo bien definido y eficiente. En realidad, el modelado de buenas prácticas replicables resulta en metodologías conocidas en el tema de Planificación Estratégica. Pero según Verweire (2014), ninguna metodología sería útil si la estrategia no se establece con claridad. Citando a él: "[...] el primer paso es acertar en el proceso de formulación. Asegurarse de que las diferentes actividades estén alineadas con la estrategia y que los colaboradores se comprometan a hacer que funcione es esencial, pero secundario". Por lo tanto, antes de cualquier aplicación de metodología o taller, Verweire (2014) menciona cuatro aspectos centrales esenciales a dominar por completo en un principio: conocimiento del mercado, el producto en sí, propuesta de valor y modelo operativo. Asimismo, es importante resaltar que la estrategia es fundamentalmente un pensamiento sobre el futuro alimentado por la información recopilada en el pasado y el presente. Pero el futuro no es lo que era y hay que considerar cualquier factor ambiental que marque la diferencia (Análisis estratégico). Estas variables son tanto de contexto nacional como internacional, y variables dentro de la organización, evidentemente. Es importante señalar que la estrategia es fundamentalmente un pensamiento sobre el futuro alimentado por la información recopilada en el pasado y el presente. Pero el futuro no es lo que era y hay que considerar cualquier factor ambiental que marque la diferencia (Análisis estratégico). Estas variables son tanto de contexto local, nacional como internacional, y las variables se encuentran dentro de la organización, evidentemente.

Por tanto, comienza el proceso de formulación. La aplicación de un análisis PEST (variables políticas, económicas, sociales y tecnológicas) podría resumir contextos tanto regionales, nacionales como internacionales. No obstante, un contexto externo fundamental también está definido por la arena competitiva. El "Modelo de las cinco fuerzas" de Porter se puede utilizar para definir la competencia directa, el poder administrado tanto por los proveedores como por los clientes y la amenaza de nuevas empresas potenciales entrantes y/o sustitutos de productos con cierta pertinencia étnica en el mercado.

Pero esta planificación es insuficiente porque una vez hecho esto, debe comenzar un pensamiento estratégico organizacional. Junto con un análisis FODA (fortalezas, debilidades, oportunidades y amenazas) para establecer el contexto relacionado con una empresa individual (Análisis estratégico), se puede definir el estado actual de los objetivos, clientes, liderazgo, control, integración, procesos y resultados. (Pensamiento estratégico). El mismo artículo también aconseja tomar estos elementos y proyectarlos dentro de tres o cinco años. 


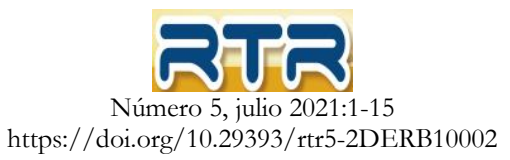

Sin embargo, como se mencionó anteriormente, el futuro es difícil de precisar, pero no imposible. Se requiere la participación de un grupo de líderes locales para resolver estas preguntas, por lo que los medios también son importantes. Hoy en día, los talleres colaborativos se convirtieron en un medio integral de los procesos de elaboración de estrategias. Se consideran $75 \%$ efectivos y ayudan a las organizaciones a mejorar su comprensión de los objetivos para el desarrollo territorial. Por último, pero no menos importante, se llevan a cabo en períodos cortos: no más de dos o tres días (Hodgkinson, 2005 et al.,). Smith \& Looman (2015) realizaron una investigación dentro de un centro de salud irlandés y destacaron la importancia de desarrollar un mapa estratégico y un cuadro de mando integral (BSC) para identificar síntomas internos para la mejora de la calidad (QI), como método alternativo. Finalmente, el método de planificación de escenarios toma lo mejor de los factores diferenciales humanos a través de la narración compartida y la creación de sentido de propósito.

Una vez que finaliza el proceso de formulación, todo líder territorial no debe olvidar estos cinco pasos cuando comienza la etapa de ejecución. Estos son: una visión holística que alcance la coordinación entre departamentos y funciones, claridad estratégica en todos los niveles antes de cualquier flexibilidad de ejecución debido a la volatilidad del mercado, encontrar la combinación correcta de coordinación, adaptación y ejecución que hará más para crear valor (utilizando correctamente el tiempo, energía y recursos territoriales), construyendo una cultura de ejecución donde todos en una organización participan e identifican buenos datos para futuros análisis y comprobaciones (Homkes, 2016).

\section{Oportunidades para el desarrollo futuro de estrategias colectivas para potenciar el impacto en las organizaciones indígenas}

La formulación de estrategias no es una actividad organizativa que deba guardarse en el cajón una vez finalizada. Es una herramienta necesaria especialmente requerida en un nuevo contexto donde el objetivo principal de los líderes territoriales y sus organizaciones tienen la visión ahora de fijar un rumbo hacia la sostenibilidad futura y de largo plazo. La visión holística planteada por Homkes (2016) podría incluso cambiar el concepto de búsqueda del beneficio de los accionistas y propietarios de negocios por el de Stakeholders, que encaja con mayor precisión en esta nueva era donde predominan la ética y los valores colectivos. Por eso la estrategia debe enfocarse en sí misma de manera colectiva.

Dicho lo anterior, cabe mencionar que la formulación de la estrategia trae consigo importantes oportunidades para las organizaciones, administraciones y grupos de interés.

En primer lugar, una estrategia bien definida podría llevar a las empresas y organizaciones locales o indígenas a una importante oportunidad para garantizar su sostenibilidad: una cultura más íntima con el cliente. Verwire (2014) concluyó que con esto se logra una relación a largo plazo donde los clientes más valiosos y rentables se mantienen leales, especialmente en sectores como el turismo cultural, la artesanía andina y empresas agrícolas indígenas. Entonces se requiere medir esto con KPI's o tener un soporte CRM. No es menos importante señalar que el $20 \%$ de los clientes generan el $80 \%$ de los ingresos (Verwire, 2014).

Otra oportunidad, a pesar de un estudio completo y eficiente de la competencia, es la posibilidad de encontrar habilidades escalables diferenciadoras actuales y potenciales, compararse con "supercompetidores", empresas con notorias ventajas competitivas, y eventualmente dar los pasos necesarios para convertirse en uno de ellos si es factible. (Hubbard et al, 2014).

Esto podría ayudar a convertir una empresa de base indígena centrada en productos locales en un negocio de plataforma en esta nueva era digital. Según Altman \& Tripsas (2013), ahora se vuelve 
imprescindible migrar de ofrecer los mejores productos a desarrollar la mejor red de complementadores, sin perder atributos o identidad originales. Un informe complementario de Birkinshaw et al (2016) ilustró la importancia de hacer alianzas estratégicas con startups y emprendimientos emergentes. Esto crea una atmósfera de intercambio de conocimientos donde se enseñan las mejores capacidades y la transición digital se vuelve más fácil para una empresa más tradicional. Los resultados finales son convincentes: la introducción de productos innovadores y el reposicionamiento tanto de la empresa tradicional como de las nuevas empresas.

Por último, todas estas oportunidades pueden conducir a la construcción de la Responsabilidad Social Corporativa (RSC), un nuevo interés especialmente reconocido por las empresas locales que se están iniciando en el mercado.

\section{Definición de la estrategia colectiva a partir de las políticas de las organizaciones que la componen, administraciones y actores claves}

Existen dos formas a través de las cuales las organizaciones son capaces de pensar en la estrategia: individual y colectivamente. La primera, corresponde a la visualización clásica de la estrategia en la que los jefes ejecutivos se encargaban de reenfocar primero sus propios lineamientos y luego comunicar los objetivos y políticas originadas a los equipos de las organizaciones y a las personas en general. El problema con esta concepción es que no considera las opiniones o sentimientos individuales de todos. Por lo tanto, se hace necesario que los líderes primero puedan identificar la propia cultura de las personas y el entorno donde viven.

En consecuencia, es importante repensar el concepto clásico de organización como entidad viva, no solo con objetivos, sino también con valores particulares y formas de proceder que se adecuen al propósito productivo y a la cultura de toda la organización.

Por lo tanto, una organización y su estrategia se forman a partir de políticas y valores traídos directamente de la cultura de las personas, y sus clientes forman parte de la misma sociedad. Anteriormente, se mencionó que el paradigma de maximizar ganancias en lugar de ayudar a la humanidad es hasta ahora un plan obsoleto de MSV (Clarke \& Friedman, 2016), pues esta misma sociedad se encarga de ayudar a los administradores a alcanzar sus metas a través de adquisiciones de gran variedad. de propuestas de valor. Esta visión se ha transformado en un punto de vista más holístico, definido por Homkes (2016), donde todos los esfuerzos para cambiar a una cultura más digna deben enfocarse en las partes interesadas.

De hecho, partes interesadas es una palabra amplia que incluye a varios socios estratégicos, externos como clientes o proveedores, e internos también. Un verdadero líder o gerente debe considerar el valor humano que las personas aportan todos los días y que les ayuda a integrarse dentro de la organización, ya sea con sus compañeros de trabajo o con sus superiores.

En segundo lugar, Peter Drucker (1954) afirma que esta nueva estrategia corporativa visualizada de manera colectiva necesita ser articulada y dinámica, tratando de unificar las ideas individuales de cada una de las áreas funcionales de la organización y el resultado sería una mejor adaptación a los ámbitos políticos, sociales y operacionales.

La propia estrategia se propone a través de varias metodologías, cuyos primeros pasos son definir el entorno donde se ubica la organización. De acuerdo con Hodgkinson et al., (2005), los talleres de estrategia son de hecho una práctica común, que a menudo involucra a gerentes muy altos y que tales 


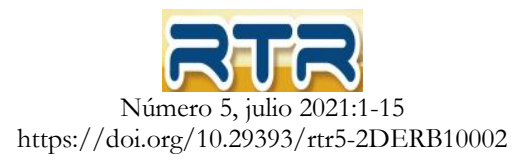

deliberaciones alimentan directamente el proceso de desarrollo de la estrategia. Entonces, por ejemplo, prácticas como el análisis PEST y / o FODA son en su mayoría elaboradas por estos ejecutivos. En conclusión, esta no es una forma colectiva de definir la estrategia. Lo que la planificación de escenarios enseña mejor es que la interacción, la narración compartida y la creación de sentido son prácticas importantes que deben inspirarse en cada miembro o equipo (Bowman, 2016). Y aquí es donde la estrategia colectiva funciona mejor que aquella en la que solo participan altos ejecutivos.

Finalmente, los líderes deben buscar las prácticas y procedimientos adecuados aplicando talleres de estrategia para que los trabajadores se sientan más iguales e involucrados en ser parte de esta construcción que definirá el futuro de la empresa. Aproximadamente el 75\% de los empleados internos informaron que los talleres proporcionaron un aporte efectivo al plan estratégico de la organización (Hodgkinson et al, 2005)

\section{Cuestiones políticas y estratégicas claves que influyen en la estrategia colectiva}

La estrategia colectiva es el resultado de varias decisiones creadas a través de un proceso metodológico que son tomadas por los participantes de una organización y su líder. Como esta vía genera consecuentemente objetivos estratégicos, lo que les da rumbo y sentido son las políticas que las organizaciones proponen para finalmente involucrar a todos en la estrategia común y cumplir con los principales objetivos.

Estas políticas deben basarse en la identidad y la diversidad. La estrategia colectiva no tendría éxito si no se alimenta de las propias capacidades de cada colaborador. De hecho, la formación de una estrategia colectiva se da gracias a una cultura organizacional que debe basarse en la diversidad.

Algunas actividades o factores clave que influyen directamente en la estrategia colectiva basada en estas políticas podrían ser:

- Coordinar la ejecución de la estrategia entre sus múltiples funciones, departamentos y unidades de la organización o comunidad.

- Comunicar explícitamente el objetivo general de la estrategia y los aspectos no negociables.

- Actuar con flexibilidad y rapidez ante las circunstancias locales y las condiciones cambiantes del mercado.

- Equilibrar actividades, recursos y la coordinación interna y adaptación a los constantes cambios externos.

- Las personas designadas como líderes de ejecución deben estar preparadas para resolver problemas, ya que tienen contacto directo con los diferentes grupos de interés.

- Mantener los compromisos y las negociaciones entre los socios de la organización y la oficina de gestión cuando las circunstancias y el entorno parecen cambiar.

- Confeccionar una historia de organización simple que comunique cuál es su camino, su competencia, cómo planea obtener los resultados y cuáles son las prioridades para que esto suceda.

- Identificar los recursos financieros, recursos humanos y tiempo que crean más valor y reasignarlos a medida que se desarrolla la situación en el ámbito local.

- Generar una cultura empresarial de aprendizaje, que tolere errores y fracasos, dando espacio a la innovación colaborativa.

- Cuestionar datos, explorar patrones ambiguos y desarrollar historias alternativas para asegurar que la ejecución de la estrategia se base en la realidad actual. 


\section{Factores políticos, globales, culturales y de interés público en el contexto global moderno}

La estrategia colectiva es un plan metodológico dentro de una organización que se sitúa en un vasto universo de factores que configuran e influyen en su propósito. Los productos obtenidos gracias a este proceso son los objetivos estratégicos, los cuales están pensados para un horizonte de largo plazo. Sin embargo, dado que este es un sentido de la palabra muy extenso, los objetivos de las principales organizaciones deben seleccionarse con precisión y cuidado de acuerdo con este vasto universo que incluye factores políticos, sociales, religiosos, mediáticos y de opinión pública.

No hay mucho que decir sobre la influencia política en la estrategia colectiva. El primer enfoque que tuvo la política dentro de una organización es el derecho mismo. Debe garantizar una serie de buenas prácticas con y entre las personas. Las organizaciones del presente se visualizan como nuevos "seres humanos", como se mencionó anteriormente según Clarke y Friedman (2016). La preocupación surge cuando el estatus quo cambia dentro de la legislación debido a intereses políticos, otra administración toma el mando o se hace evidente que la ley tiene que evolucionar. En consecuencia, es evidente que las organizaciones deben adecuar su piel para detectar los factores políticos en el presente y aplicar metodologías estratégicas, ganando la capacidad de ser lo suficientemente eficientes para determinar factores que podrían cambiar los escenarios políticos y legales, de manera directa y no directa. relacionados con su propio contexto.

Los factores ambientales no son exclusivos de la propaganda social. La evidencia científica ha demostrado que el cambio climático global es un hecho, por lo que también se convirtió en una preocupación política. Por lo tanto, la ley está evolucionando para ser un mejor brazo de protección ambiental, por lo que aspectos como la sostenibilidad se vuelven imprescindibles para ser incluidos en la estrategia corporativa, no solo para cumplir con los requisitos legales sino también para la opinión pública. Al final, el público en general son clientes potenciales.

Hablando de influencia global, no es ningún secreto que las fronteras se están volviendo restrictivas solo en el marco legal a medida que el mundo se vuelve más digitalizado. La digitalización es la consecuencia global más importante de los últimos años. El episodio pandémico causado por el COVID-19 ha logrado acelerar este proceso en unos pocos meses.

Algunas páginas web conocidas como eBay o PayPal fueron creadas directamente como negocios basados en plataformas, y otras que no lo hicieron, están participando en este fenómeno tratando de cambiar su cultura y procesos sin perder su identidad.

El impacto cultural y mediático están relacionados entre sí. Es fundamental conocer el círculo cultural donde una organización planea nacer desde el principio y también hacer un seguimiento de los cambios que puedan surgir posteriormente. Las preferencias culturales de las personas son un denominador común que generalmente surge de brechas generacionales y ubicaciones geográficas. Los medios y la religión son manifestaciones de estos.

Entonces, cuanto más una organización conozca a las personas que rodean sus operaciones, mejor será la opinión pública o incluso las ganancias que obtendrán. Willis (2015) define que la Responsabilidad Social Corporativa (RSC) está representada a través de cuatro áreas clave: el medio ambiente, el patrimonio, el mercado y la comunidad. El mercado y la comunidad están relacionados: el mercado se basa en personas de una determinada comunidad y las preferencias y la cultura de la comunidad definen 
las características principales del mercado. Este es un paso importante para tener en cuenta al pensar en la estrategia colectiva con todos los miembros de la organización.

\section{Cómo se pueden resolver las tensiones éticas de las organizaciones, las administraciones y las partes interesadas clave}

Generalmente se pueden encontrar discrepancias entre cómo se enseña la cultura desde que alguien es pequeño y crece y cómo la ética tiene la capacidad de cambiar lo que culturalmente parecía normal. Y lo que puede ser útil para una organización es identificar el conjunto de valores que ambos tienen en común y, en consecuencia, elaborar una serie de buenas prácticas que deben estar impregnadas de culturales y éticas. Aun así, estas prácticas son fundamentales para establecer la fidelidad de los clientes y una opinión pública no deficiente. Clarke y Friedman (2016) detallaron nueve costumbres poco éticas que deben evitarse de la antigua cultura de maximizar el valor para los socios y, por lo tanto, qué prácticas deben compartirse en las políticas estratégicas:

- Mantener datos transparentes y confiables en los estados financieros compartidos por contadores o lideres de la organización.

- Priorizar la inversión de capital destinada a más empleo y mayores beneficios para todos los emprendimientos u organizaciones indígenas.

- Buscar la satisfacción de los miembros de la organización, incluso si esto incluye repensar un producto o servicio que podría dañar el patrimonio local o la conservación del entorno medioambiental.

- Esta satisfacción debe planificarse a largo plazo, haciendo de la fidelidad de los grupos de interés una prioridad.

- Una organización que paga sus impuestos y cuotas sociales la hace responsable en el sentido de que la inversión es beneficiosa para las personas y la creación de mejores condiciones para la organización y su territorio.

- Adicionalmente, debe elaborar y actualizar políticas ambientales dentro de sus procesos, buscando mantener su huella de carbono lo más baja posible.

- Contratación de líderes indígenas con alta innovación, creatividad y excelente experiencia en temas patrimoniales y desarrollo económico local.

\section{Inteligencia estratégica en el desarrollo y formulación de la estrategia}

La inteligencia estratégica es el corazón de la planificación estratégica, porque, aunque esta segunda conlleva metodologías que ayudan a moldear los objetivos y planes a largo plazo que se encuentran en la concepción de la estrategia, la inteligencia estratégica brinda un insumo importante sobre cómo se debe dar forma a esta estrategia, donde el estatus quo ya no es una constante, donde la nueva constante es el cambio y donde los problemas son bienvenidos en lugar de rechazados. Incluso se espera estimular la solución de antiguos y nuevos problemas locales para plantear retos que lleven a la organización por un nuevo camino.

Como es de conocimiento común, las estrategias son diseñadas, ejecutadas y recibidas por hombres y mujeres con características y necesidades variadas. Aunque estos individuos cumplen diferentes roles en la sociedad, no actúan de la misma manera en todos los contextos. El denominador común que unifica esta diversidad para una planificación estratégica inteligente es el propósito. La diversidad es esencial si se planea una estrategia colectiva porque, no obstante, volvería a ser una estrategia de altos directivos. 


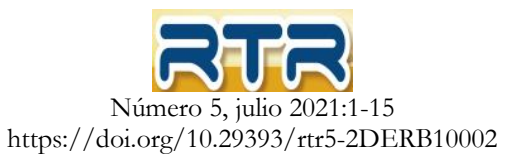

La palabra propósito conlleva un proyecto común que vincula los bienes materiales y la designación de tareas productivas para el logro de metas.

En este contexto, la identidad, el compromiso, la confianza y la lealtad se hacen necesarias para abrirse a todas las posibilidades que surgen y conforman la estrategia.

La inteligencia estratégica se basa en estos factores para encontrar una herramienta de éxito (Pensamiento estratégico):

- Primero, es necesario atreverse a explorar lo desconocido en cuanto a mejoras, satisfacción de los socios y cambios futuros. La satisfacción del usuario es un pilar de todo modelo de negocio, sin olvidar mencionarlo.

- En segundo lugar, cada integrante de la organización debe asumir que el proceso de cambio y adaptación es constante, porque si no hay problemas, como se dijo primero, hay que descubrirlos para generar soluciones y, efectivamente, nuevas propuestas desafiantes.

- En tercer lugar, se debe animar a todos a comunicar sus pensamientos y reflexiones y a ser capaces de aceptar una recepción positiva o negativa. No es menos importante reconocer a los líderes más experimentados con el mismo pensamiento de siempre, al ritmo que se crean nuevas voces frescas.

- Cuarto, todas las personas tienen que reconocer que nadie es dueño de la verdad, porque en una organización o comunidad todas las visiones nutren el sentido común que da vida a la estrategia.

- Quinto, el aprendizaje y el desarrollo constante son el corazón de una organización estratégica.

Por tanto, la inteligencia estratégica aplicada al desarrollo y formulación de una estrategia organizacional es útil para el desarrollo interpersonal en un clima que propicia el intercambio de ideas con respeto, empatía y confianza.

Gracias a este próximo paso se espera que sea el diálogo y la construcción de una historia común en la que todos participarán y avanzarán para alcanzar una acción estratégica. En consecuencia, esta historia será transmitida, tanto a los miembros de la organización como a las entidades públicas, quienes harán un aporte participando de la planificación de un escenario futuro.

\section{Conocimiento y pensamiento original para la formulación y desarrollo de la estrategia colectiva}

La práctica de la planificación de escenarios como herramienta estratégica conecta la esencia distintiva del ser humano (razonar, comunicar y socializar), conceptualizándolo como una práctica de la simplexidad, donde la complejidad del pensamiento se conjuga con la simplicidad de la acción, necesaria para comprender y transmitir la complejidad. que rodea los procesos organizacionales (Bowman, 2016). En otras palabras, la planificación de escenarios no es más que un proceso comunicativo y la narración un acto de habla. 


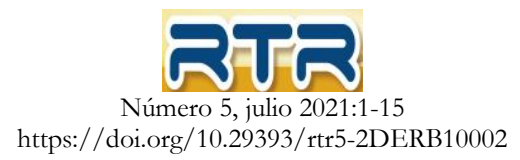

Esta simplexidad ayuda a dar forma a dos resultados de este método: el proceso y los escenarios. Los procesos internos donde la mayoría de las personas tienen un espacio son discursivos, episódicos y prácticas que combinan la creación de sentido, la organización y la narración. Los escenarios son producto de este mismo proceso, donde se identifican problemas y por lo tanto se diseñan nuevas prácticas para transmitir fácilmente esta conceptualización: los escenarios. Son objetos de frontera que se transfieren como conocimiento a través de otras fronteras estratégicas (Bowman, 2016).

La forma mejor aceptada de comunicar la complejidad de los escenarios es la narración de historias, y se ha citado anteriormente que se trata de una práctica excelente para la inteligencia estratégica, en la que se descubre que todos los interesados tienen una historia que contar y que crearía valor en un modelo de negocio colaborativo. La narración de historias no es una práctica ligera como parece, porque se sustenta en una gran creación de sentido de propósito, de una manera interorganizacional.

La planificación de escenarios es una actividad estructurada, analítica y similar a un embudo donde su principal objetivo es la creación de múltiples futuros para ayudar a las partes interesadas a volver a percibir la realidad y así mejorar las decisiones estratégicas o políticas. Bowman (2016) aporta lo mejor de la socialización de los retos de la organización o comunidad a través de talleres participativos.

\section{Diferencias entre los objetivos de la estrategia organizacional individual y la estrategia colectiva}

A pesar de que la estrategia es casi un concepto nuevo introducido recientemente en la gestión organizacional, ciertamente se ha difundido más en la formación de gerentes generales y ejecutivos de alto rango.

Por lo tanto, el propósito principal de la estrategia individual es mejorar los objetivos de las empresas originales, desarrollando las ideas de los gerentes y fundadores. Se sabe que toda organización nace con objetivos basados en los valores culturales del emprendedor. El error es creer que esto debería permanecer estático durante un largo período de tiempo. Sí, es correcto asimilar la idea de que una empresa no es solo una entidad que produce riqueza para sus fundadores, sino un cuerpo vivo con ética, valores y cultura porque los seres humanos son parte de ella, como se indicó anteriormente en los párrafos anteriores. En realidad, el problema sigue siendo pensar que esta conjunción de pensamientos, ideas e inspiraciones debería ser la misma para siempre. La inteligencia estratégica es una ventaja que surge con el propósito de romper este paradigma de una vez por todas,

Convencionalmente, el único paso que se daba en la formulación de la estrategia por parte de los líderes y altos directivos era elaborar un análisis eficiente aplicando los métodos explicados anteriormente, mirando el entorno y las propias fortalezas y debilidades de las organizaciones (Análisis estratégico). Sin embargo, incluso el mejor y más completo análisis es insuficiente si no hay intención de cambiar al pensamiento estratégico en el proceso. Líneas arriba, la inteligencia estratégica se definió como un intento de romper el hielo y probar lo nuevo, abrir la mente e incluso crear los problemas para nuevas soluciones sin precedentes (Pensamiento estratégico) en un mundo que cambia continuamente.

Con todo esto dicho, se afirma de manera indirecta pero correcta que aprender del entorno es fundamental para la planificación y aplicación de la estrategia individual. De hecho, no hay más opción: se debe poder transformar su organización en una piel poderosa que constantemente detecte cambios externos, clasifique y almacene los más importantes para recursos estratégicos y, en consecuencia, genere conclusiones que evidentemente conducirán a aprender de ellos. 


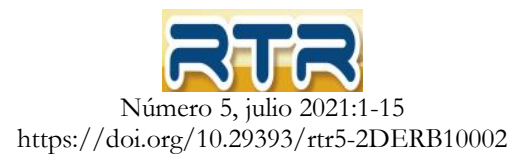

El propósito de la estrategia individual es encontrar la manera correcta de reinventar el enfoque personal utilizando la estimulación de la mentalidad abierta, lo necesario que una organización necesita para innovar. Esto está íntimamente relacionado con cómo los empleados administran su tiempo, cómo son recompensados, entre otros. Sin embargo, la principal debilidad de la estrategia individual es que, a pesar de las buenas intenciones, sigue siendo personal y converge en unas pocas entidades. Eso significa conflicto, porque algunas personas prefieren permanecer en su zona de confort y aplicar la ley del mínimo esfuerzo.

Es por esto por lo que surge la estrategia colectiva, inclusiva con todos los miembros de una organización y cuyo propósito es aprovechar las capacidades y disposición individuales para generar sentido común, que incentive a las personas a progresar.

Este sentido generará una simbiosis organizativa, donde los medios de comunicación serán el diálogo, la narración y la transfiguración de lo complejo en términos más sencillos y atractivos. Así, se espera proyectar resultados grupales, que se convertirían en acciones y finalmente en realidades.

\section{Aspiraciones de la estrategia colectiva y los objetivos éticos, culturales y operativos de las organizaciones}

Como la estrategia colectiva se fundamenta bajo la convicción de que las habilidades individuales construyen una estrategia más consolidada, tomando lo mejor de cada integrante y con la predisposición de aprender de los cambios constantes, es cierto afirmar que sus aspiraciones surgen de la red de relaciones sociales que día a día une valores individuales y ayuda a las personas a aprender unas de otras. Sí, es importante ser sensible al exterior, pero es fundamental serlo internamente con otros compañeros que representan también una fuente de conocimiento. Eventualmente, un espacio donde todos puedan compartir ideas es beneficioso para realizar estudios y análisis externos profundos. Esto también está vinculado a los objetivos éticos, culturales y operativos de las organizaciones y las partes interesadas.

Por tanto, la estrategia colectiva está definitivamente influenciada por los pensamientos, sentimientos, valores y decisiones de las personas que forman parte de la empresa u organización. Cabe destacar nuevamente que una organización se constituye como un ente vivo, con objetivos, políticas, creencias, una cultura, valores particulares y formas de proceder. Todos estos rubros deben ser alistados, conocidos vertical y horizontalmente y adaptados al propósito productivo de la cadena de valor.

Así, una organización exitosa consolida una cultura social y ética que es integral y logra representar a cada persona individual que forma parte de las áreas operativas de la firma, por diferentes que sean.

A través de todo este proceso, la construcción de una historia común se volvería real, donde la participación sería común tanto en la planificación como en la ejecución de la estrategia. En cualquier caso, una cultura bien constituida sin duda conducirá a una propuesta de valor que garantizará la satisfacción de los diferentes grupos de interés. Los consumidores también forman parte de un entorno cultural, por lo que podrían mostrar empatía hacia esta nueva visión fresca de los negocios indígenas.

\section{Conclusiones}

El propósito principal de este artículo fue explorar la teoría y la práctica de la estrategia colectiva en las organizaciones que anhelen mantenerse sostenibles, ya que preocuparse solo en el "valor" para los 


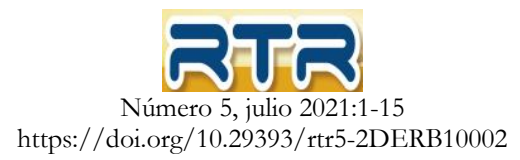

propietarios de negocios y líderes de organizaciones indígenas locales es insuficiente. El mundo está cambiando rápidamente, por lo que las nuevas teorías, pensamientos y objetivos deben desarrollarse en el largo plazo, ya que los primeros aspectos mencionados pertenecen al corto y mediano plazo.

Todas estas actividades requieren un cambio de paradigma urgente, porque no sólo se basan en herramientas metodológicas, como el Cuadro de Mando Integral, FODA, PESTEL o Planificación de Escenarios. De hecho, Escenario Planning establece los asentamientos para una planificación estratégica más inteligente. El Pensamiento Estratégico necesita en consecuencia un enfoque crítico diferente en las dimensiones organizativa, filosófica y metodológica, diferente de los talleres de estrategia participativa común. Además, se considera el cambio como la única constante, y la innovación y participación respaldan esta afirmación. Esto conducirá a mejores capacidades para sobrevivir en un entorno económico, político, social y tecnológico cada vez más competitivo, al tiempo que se construyen los objetivos y recursos para enfrentar los desafíos locales.

En este contexto, el liderazgo en la estrategia colectiva requiere dos consideraciones principales que podrían resumir todo el ensayo, una a nivel gerencial y otra a nivel organizacional. El primero considera que se requieren líderes abiertos a la innovación y a nuevas ideas. Líderes territoriales que sean capaces de distribuir su tiempo para atender necesidades urgentes como: planificar actividades de desarrollo, construir relaciones, recibir retroalimentación honesta sobre la forma en que se realiza el trabajo, concentrarse en el desarrollo colectivo y explorar nuevas mejoras, iteraciones o modificaciones a la propuesta de valor local.

La segunda es la planificación estratégica, la cual actuaría bajo una lógica narrativa que permitirá un flujo de información simple e inmediato, para las actividades de toma de decisiones exitosas y para un correcto despliegue de la estrategia organizacional, en tiempo real y siguiendo estos principios: una visión holística, claridad estratégica en todos los niveles, encontrando la combinación correcta de coordinación, adaptándose a las acciones para crear valor, construyendo una cultura de ejecución donde todos en una organización participen (Homkes, 2016).

Finalmente, el artículo al explorar la importancia de la estrategia colectiva para apoyar el desarrollo indígena Aymara, corresponde en todo caso a la actual visión de la marginalización del contexto indígena dentro del sistema global. En tal sentido, la visión convencional del desarrollo, como proceso principalmente reducido a elevar la productividad mediante el progreso técnico, inspirados desde estructuras institucionales externas, no son necesariamente efectivas y respetuosas con las estrategias colectivas locales. En otras palabras, y tal como lo establece el Dr. José Luis Sampedro (2019, pp. 398), "La acción sobre las estructuras locales, para hacerlas evolucionar a lo largo de la dimensión del cambio social es indispensable para un auténtico desarrollo, exigir un camino alternativo", asentado en una estrategia colectiva con mayor participación y compromiso de los agentes económicos e institucionales del territorio.

\section{Referencias}

Abolafia, MY (2010). 'La construcción narrativa como construcción de sentido: cómo piensa un banco central', Organization Studies, 31, págs. 349-367.

Altman y Tripsas (2013). "Cómo convertir una empresa centrada en productos en una plataforma empresarial"

Barbero (2005). "Enfoque político de la estrategia" 
Barra, RIN. 2018. Casos de estudio de centros de desarrollo empresarial en Chile y comunidades indíges Aymaras. Primer Congreso de Emprendimiento y Desarrollo Local. Concepción, Chile.

Bowman (2016). "La práctica de la planificación de escenarios: un análisis de la elaboración de estrategias inter e intraorganizacionales"

Birkinshaw, Robbins \& Ryan (2016) "Cómo familiarizarse con la transformación digital"

Clarke y Friedman (2016). "Maximizar el valor para los accionistas: una teoría enloquecida"

Gary Bowman. La práctica de la planificación de escenarios: un análisis de la elaboración de estrategias inter e intraorganizacionales, British Journal of Management, vol. 27, 77-96 (2016)

Chandler, A. 1962. Estrategia y estructura. The MIT Press, Cambridge, MA.

Drucker, P 1954. La práctica de la gestión. Harper, Nueva York.

Hoffman (2014). "Estrategias que las empresas están utilizando para mitigar y adaptarse al cambio climático"

Hodgkinson, Johnson, Whittington \& Shwarz (2005) "El papel y la importancia de los talleres de estrategia: Hallazgos de una encuesta del Reino Unido"

Homkes (2016). "Traducir la estrategia en resultados"

Hubbard, Leinwand y Mainardi (2014). "Lo que su empresa puede aprender de los supercompetidores"

Kourdi (2010). "El futuro no es lo que solía ser"

178-6

José Luis Sampedro (2019) “Economía Humanista; Algo más que cifras”. ISBN: 978-84-9908-

Smith y Looman (2015) "Explorando la ejecución estratégica. Un estudio de caso sobre el uso del cuadro de mando integral en un hospital irlandés "

Kenneth A. 1971. El concepto de estrategia corporativa. Irwin, Homewood, III. Meabh Smith, John Loonam, (2016) "Exploración de la ejecución estratégica: un estudio de caso sobre el uso del cuadro de mando integral en un hospital irlandés", Journal of Strategy and Management, vol. 9 Edición: 4, págs. 406-428

Meabh Smith, John Loonam, (2016) "Exploración de la ejecución estratégica: un estudio de caso sobre el uso del cuadro de mando integral en un hospital irlandés", Journal of Strategy and Management, vol. 9 Edición: 4, págs. 406-428.

Van Marrewijk (2016). "Subculturas en conflicto en fusiones y adquisiciones: un estudio longitudinal de la integración de una empresa de Internet radical en una empresa de telecomunicaciones burocrática"

Verweire (2014). "Cómo ser una empresa íntima con el cliente"

Verweire (2014). "Cómo formular una estrategia ganadora"

Willis (2015). "El ABC de la RSE. Los programas de responsabilidad social corporativa son fáciles de equivocar”. 